\title{
AN ESTIMATE ON THE VOLUME OF METRIC BALLS
}

\author{
BY SHIGERU KODANI
}

\section{Introduction.}

Let $M$ be a complete Riemannian manifold of dimension $n$. We denote by $i(M)$ the injectivity radius of $M$, by $B(p, r)$ the metric ball in $M$ of radius $r \leqq$ $i(M)$ centered at $p \in M$ and by $\operatorname{vol}(B(p, r))$ the volume of $B(p, r)$. Furthermore we denote by $\alpha(n)$ the volume of the round sphere $S^{n}$ of sectional curvature 1 . M. Berger and J. Kazdan [3] showed that if $M$ is closed then the volume $\operatorname{vol}(M)$ of $M$ satisfies

$$
\operatorname{vol}(M) \geqq \alpha(n)(i(M) / \pi)^{n},
$$

where the equality holds if and only if $M$ is a round sphere of constant sectional curvature $(\pi / i(M))^{2}$. Later, C. B. Croke [6] showed that if $M$ is closed then for $r \in[0, i(M)]$,

$$
\underset{x \in M}{\operatorname{Ave}} \operatorname{vol}(B(x, r)) \geqq \alpha(n)(r / \pi)^{n} .
$$

Here the equality holds if and only if $r=i(M)$ and $M$ is a round sphere. Here $\underset{x \in M}{\operatorname{Ave}} f(x)$, for any function $f$ on $M$, means $\frac{1}{\operatorname{vol}(M)} \int_{M} f(x) d x$. But it is believed that for any point $p \in M$ and for $r \in[0, i(M)]$,

$$
\operatorname{vol}(B(p, r)) \geqq \alpha(n)(r / \pi)^{n} .
$$

Here the equality holds if and only if $r=i(M), B(p, i(M))=M$ and $M$ is a round sphere. As partial results on this problem, not sharp lower bounds are already known ([1], [2] for $n=2,3$ and [4] for all $n$ ). And under some restriction on the metric form, a sharp one is obtained by C. B. Croke [5]. Especially C. B. Croke [4] showed the following remarkable inequality,

$$
\operatorname{vol}(B(p, r)) \geqq\left[\frac{\pi \alpha(n-1)}{n \alpha(n)}\right]^{n} \alpha(n)\left[\frac{r}{\pi}\right]^{n} .
$$

Here

$$
\left[\frac{\pi \alpha(n-1)}{n \alpha(n)}\right]^{n} \approx\left[\frac{\pi}{2 n}\right]^{n / 2}, \quad n \rightarrow \infty .
$$

Received March 24, 1998 
In this paper, we will improve the Croke's constant under an additional curvature condition on the metric balls.

\section{Result.}

Let $k$ be the infimum of the sectional curvature of the metric ball $B(p, r)$ in a complete Riemannian manifolds $M$. If the radius $r$ is not greater than the injectivity radius $i(M)$ of $M$, then by Myers' theorem (cf. [7]), we see $k \leqq$ $(\pi / r)^{2}$, and by maximal diameter theorem (cf. [7]), the equality holds if and only if $r=i(M), \quad M=B(p, i(M))$ and $M$ is a round sphere. Our result is the following.

THEOREM. Let $B(p, r)$ be a metric ball of radius $r$ centered at $p$ in a complete Riemannian manifold $M$. Let $k$ be the infimum of the sectional curvature of the metric ball $B(p, r)$. Then, for $r \leqq i(M)$, there exist an increasing function $f:\left[-\infty,(\pi / r)^{2}\right] \rightarrow[0,1]$ such that

$$
f\left((\pi / r)^{2}\right)=1, \quad f(0)>(2 / 3)^{n}, \quad f(-\infty)=0
$$

and

$$
\operatorname{vol}(B(p, r)) \geqq \frac{(n+3)}{6(n+1)} f(k) \alpha(n)\left[\frac{r}{\pi}\right]^{n}
$$

Before we prove the theorem, we need some definitions. For $x \in B(p, r)$, put

$$
E(x, r)=\exp _{x}^{-1}(B(x, r) \cap B(p, r)),
$$

and define $\operatorname{vol}(E(x, r))$ as the euclidiean volume in the tangent space $T_{x} M$ at $x$. As a special case of the inequality in theorem $A$ of [6], we get

$$
\underset{x \in B(p, r)}{\operatorname{Ave}} \operatorname{vol}(B(x, r) \cap B(p, r)) \geqq \frac{(n+3) \alpha(n)}{6(n+1) \pi^{n} \beta(n)} \cdot \underset{x \in B(p, r)}{\operatorname{Ave}} \operatorname{vol}(E(x, r)),
$$

where $\beta(n)$ is the volume of the standard disk of radius 1 in Euclidiean space $R^{n}$. Evidently,

$$
\operatorname{vol}(B(p, r)) \geqq \underset{x \in B(p, r)}{\text { Ave }} \operatorname{vol}(B(x, r) \cap B(p, r)) .
$$

Let $M_{k}$ be an $n$-dimensional simply connected space form of sectional curvature $k$ and $B_{k}(q, r)$ be a metric ball in $M_{k}$ at $q \in M_{k}$. Then the following lemma holds.

LEMMA. For all $p \in M, q \in M_{x}$ and $0<r \leqq i(M)$,

$$
\underset{x \in B(p, r)}{\operatorname{Ave}} \operatorname{vol}(E(x, r)) \geqq \underset{y \in B_{k}(q, r)}{\operatorname{Ave}} \operatorname{vol}\left(E_{k}(y, r)\right) .
$$


Proof. Fix an isometry $I_{p}: T_{p} B(p, r) \rightarrow T_{q} B_{k}(q, r)$. For $x \in B(p, r)$, we put $y=\exp _{q} \circ I_{p} \circ \exp _{p}^{-1}(x)$ and put $s=d(p, x)=d_{k}(q, y)$, where $d\left(d_{k}\right.$, resp.) is the distance on $B(p, r)\left(B_{k}(q, r)\right.$, resp.). Then we get an isometry $I_{x}=\tau_{q}^{y} \circ I_{p} \circ \tau_{x}^{p}$ : $T_{x} B(p, r) \rightarrow T_{y} B_{k}(q, r)$, where $\tau_{x}^{x}$, is the parallel translation from $T_{x^{\prime}} M$ to $T_{x} M$. For a unit vector $v \in T_{x} M$, let $l(v)$ denote the length of the geodesic segment $\gamma_{v}$ emanating from $x$ with the velocity vector $\dot{\gamma}(0)=v$ and hitting the boundary of $B(p, r) \cap B(x, r)$ at $\gamma_{v}(l(v))$. Similarly for $v^{\prime}=I_{x}(v) \in T_{y} M$, we define $\gamma_{v^{\prime}} \subset$ $B(q, r)$ and $l\left(v^{\prime}\right)$.

Let $\xi_{x} \subset B(p, r)\left(\xi_{y} \subset B_{k}(q, r)\right.$, resp. $)$ be the distance minimizing geodesic segment from $p$ ( $q$, resp.) to $x$ (y, resp.). Since $I_{x}$ is isometry, we see

$$
\left\langle v,-\dot{\xi}_{x}(s)\right\rangle=\left\langle v^{\prime},-\dot{\xi}_{y}(s)\right\rangle \text {. }
$$

By Toponogov's triangle comparison theorem (cf. [7]), we obtain

$$
d\left(p, \gamma_{v}(t)\right) \leqq d_{k}\left(q, \gamma_{v^{\prime}}(t)\right)
$$

for all $0 \leqq t \leqq \min \left(l(v), l\left(v^{\prime}\right)\right)$. Therefore if $l(v)<r$ then $\gamma_{v}$ hits the boundary of $B(p, r)$, and so,

$$
d\left(p, \gamma_{v}(l(v))\right)=d_{k}\left(q, \gamma_{v^{\prime}}\left(l\left(v^{\prime}\right)\right)\right)=r .
$$

On the other hand, if $l(v)=r$ then $l\left(v^{\prime}\right) \leqq r=l(v)$. Therefore we always find

$$
l(v) \geqq l\left(v^{\prime}\right),
$$

and so,

$$
\begin{aligned}
\operatorname{vol}(E(x, r)) & =\int_{0}^{l(v)} \int_{S^{n-1}} r^{n-1} d r d v=\int_{S^{n-1}} \frac{l(v)^{n}}{n} d v \\
& \geqq \int_{S^{n-1}} \frac{l\left(v^{\prime}\right)^{n}}{n} d v^{\prime}=\operatorname{vol}\left(E_{k}(y, r)\right):=V(r) .
\end{aligned}
$$

Evidently, $d V / d s(s)=\dot{V}(s)<0$.

By Bishop's inequality (cf. [8]), we have, for $0 \leqq s \leqq s^{\prime} \leqq r$,

and by (11), we get

$$
\frac{\operatorname{vol}(B(p, s))}{\operatorname{vol}\left(B\left(p, s^{\prime}\right)\right)} \geqq \frac{\operatorname{vol}\left(B_{k}(q, s)\right)}{\operatorname{vol}\left(B_{k}\left(q, s^{\prime}\right)\right)},
$$

$$
\begin{aligned}
\underset{x \in B(p, r)}{A v e} \operatorname{vol}(E(x, r)) & =\frac{\int_{B(p, r)} \operatorname{vol}(E(x, r)) d x}{\operatorname{vol}(B(p, r))} \geqq \frac{\int_{0}^{r} \int_{\partial B(p, s)} V(s) d s d x}{\operatorname{vol}(B(p, r))} \\
& =\frac{\int_{0}^{r} \operatorname{vol}(\partial B(p, s)) V(s) d s}{\operatorname{vol}(B(p, r))} \\
& \geqq\left[\frac{\operatorname{vol}(B(p, s)) V(s)}{\operatorname{vol}(B(p, r))}\right]_{0}^{r}-\frac{\int_{0}^{r} \operatorname{vol}(B(p, s)) \dot{V}(s) d s}{\operatorname{vol}(B(p, r))}
\end{aligned}
$$




$$
\begin{aligned}
& \geqq\left[\frac{\operatorname{vol}\left(B_{k}(q, s)\right) V(s)}{\operatorname{vol}\left(B_{k}(q, r)\right)}\right]_{0}^{r}-\frac{\int_{0}^{r} \operatorname{vol}\left(B_{k}(q, s)\right) \dot{V}(s) d s}{\operatorname{vol}\left(B_{k}(q, r)\right)} \\
& =\underset{y \in B_{k}(q, r)}{\operatorname{Ave}} \operatorname{vol}\left(E_{k}(y, r)\right) .
\end{aligned}
$$

Proof of Theorem. Put

$$
f(k)=\frac{1}{\beta(n) r^{n}} \underset{y \in B_{k}(q, r)}{\operatorname{Ave}} \operatorname{vol}\left(E_{k}(y, r)\right) .
$$

We can easily verify that $f$ is strictly increasing and $f(\pi / r)^{2}=1, f(-\infty)=0$. By combining (7) with (8) and (9), we get (6). $f(0)$ is calculated as follows:

$$
\begin{aligned}
f(0)= & \frac{1}{\beta(n)} \underset{y \in B_{0}(q, 1)}{\operatorname{Ave}} \operatorname{vol}\left(E_{0}(y, 1)\right) \\
= & \frac{1}{\beta(n)^{2}} \int_{0}^{1} \alpha(n-1) r^{n-1} 2 \int_{r / 2}^{1} \beta(n-1)\left(1-s^{2}\right)^{n-1 / 2} d s d r \\
= & \frac{2 \alpha(n-1) \beta(n-1)}{\beta(n)^{2}}\left\{\int_{0}^{1} r^{n-1} \int_{1 / 2}^{1}\left(1-s^{2}\right)^{n-1 / 2} d s d r\right. \\
& \left.\quad+\int_{0}^{1} r^{n-1} \int_{r / 2}^{1 / 2}\left(1--s^{2}\right)^{n-1 / 2} d s d r\right\} .
\end{aligned}
$$

Here we have

$$
\int_{0}^{1} r^{n-1} \int_{1 / 2}^{1}\left(1-s^{2}\right)^{n-1 / 2} d s d r=\frac{1}{n} \int_{0}^{\pi / 3} \sin ^{n} \vartheta d \vartheta
$$

By Fubini's theorem, we have

$$
\begin{aligned}
\int_{0}^{1} r^{n-1} \int_{r / 2}^{1 / 2}\left(1-s^{2}\right)^{n-1 / 2} d s d r & =\int_{0}^{1 / 2}\left(1-s^{2}\right)^{n-1 / 2} \int_{0}^{2 s} r^{n-1} d r d s \\
& =\frac{1}{n} \int_{0}^{1 / 2}\left(1-s^{2}\right)^{n-1 / 2}(2 s)^{n} d s \\
& =\frac{1}{n} \int_{\pi / 3}^{\pi / 2}(2 \sin \vartheta \cos \vartheta)^{n} d \vartheta \\
& =\frac{1}{2 n} \int_{2 \pi / 3}^{\pi} \sin ^{n} \vartheta d \vartheta=\frac{1}{2 n} \int_{0}^{\pi / 3} \sin ^{n} \vartheta d \vartheta
\end{aligned}
$$

Hence, we get

$$
\begin{aligned}
f(0) & =\frac{3 n}{n-1} \frac{\alpha(n-2)}{\alpha(n-1)} \int_{0}^{\pi / 3} \sin ^{n} \vartheta d \vartheta \\
& >\frac{3 n}{n-1} \frac{\alpha(n-2)}{\alpha(n-1)}\left\{\frac{1}{\alpha(n)} \frac{\alpha(n+1)}{2}\left(\frac{2 \pi / 3}{\pi}\right)^{n+1}\right\}=\left(\frac{2}{3}\right)^{n}
\end{aligned}
$$


where we have used the relations

$$
n \beta(n)=\alpha(n-1), \quad \alpha(n+2)=2 \pi \alpha(n) /(n+1),
$$

and replaced the volume of the spherical cup of radius $\pi / 3$ in the unit $(n+1)$ sphere by the volume of the hemisphere of $(n+1)$-dimensional round sphere with diameter $2 \pi / 3$.

q.e.d.

Remark. Put

Table 1.

\begin{tabular}{rcc}
\hline$n$ & $c_{1}(n)$ & $c_{3}(n)$ \\
\hline 2 & .616849 & .1234568 \\
3 & .296296 & .0740741 \\
4 & .120394 & .0460905 \\
5 & .043151 & .0292638 \\
6 & .013989 & .0188125 \\
7 & $4.17219 \times 10^{-3}$ & .0121933 \\
10 & $7.45077 \times 10^{-5}$ & $3.41576 \times 10^{-3}$ \\
15 & $3.48113 \times 10^{-8}$ & $4.28182 \times 10^{-4}$ \\
$\cdots$ & $\cdots$ & $\cdots$ \\
$n \rightarrow+\infty$ & $\approx\left[\frac{\pi}{2 n}\right]^{n / 2}$ & $\approx \frac{1}{6}\left[\frac{2}{3}\right]^{n}$ \\
\hline
\end{tabular}

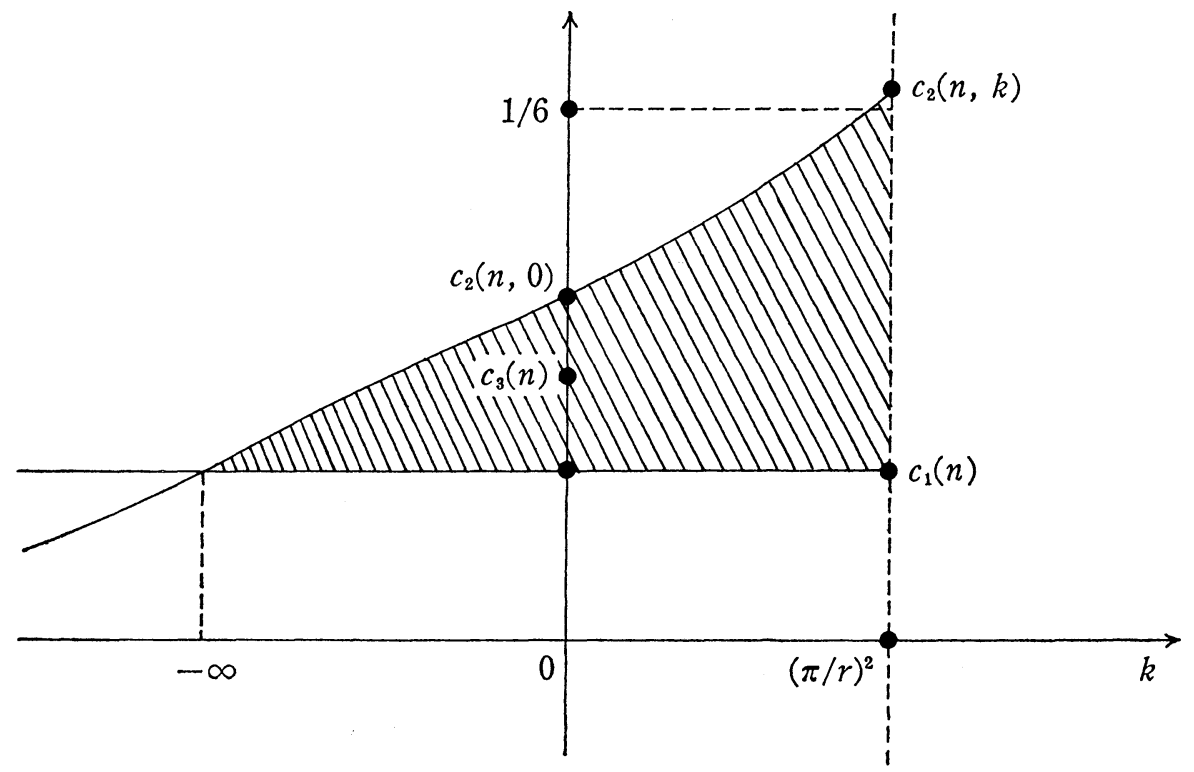

Fig. 2. 


$$
\begin{gathered}
c_{1}(n)=\left[\frac{\pi \alpha(n-1)}{n \alpha(n)}\right]^{n}, \quad c_{2}(n, k)=\frac{(n+3)}{6(n+1)} f(k), \\
c_{3}(n)=\frac{(n+3)}{6(n+1)}\left(\frac{2}{3}\right)^{n} .
\end{gathered}
$$

Then we have $c_{2}(n, 0)>c_{3}(n)$. Now we give the explicit values of $c_{1}(n)$ and $c_{3}(n)$ in Table 1 . From this table, we can observe that if the sectional curvature $k$ of the metric ball is positive then, for $n \geqq 6$, our constant $c_{2}(n, k)$ is better than Croke's constant $c_{1}(n)$ (See also Fig. 2.).

\section{REFERENCES}

[1] M. BERger, Some relations between volume, injectivity radius, and convexity radius in Riemannian manifolds. Differential Geometry and relativity (Cahen, Flato, Eds.), D. Reidel, Dordrecht-Boston, 1976.

[2] M. BERger, Volume et rayon d'injectivite dans les varietes riemanniennes de dimension 3, Osaka J. Math. 14 (1977), 191-200.

[3] M. BERGER AND J.L. KAZDAN, A Sturm-Liouville inequality with applications to an isoperimetric inequality for volume in terms of injectivity radius, and to Wiedersehen manifolds, in General inequalities 2 (Proceedings of Second International Conference on General Inequalities, 1978), E.F. Beckenbach (ed.), ISNM47, 367-377, Basel, Birkhauser 1980.

[4] C. B. Croke, Some isoperimetric inequalities and Eigenvalue estimates, Ann. Sci. Ecole Norm. Sup. (4) 13 (1980), 419-535.

[5] C. B. CROKE, On the volume of metric balls, Proc. Amer. Math. Soc. 88 (1983), 660-664.

[6] C.B. CROKE, Curvature free volume estimates, Invent. math. 76 (1984), 515-521.

[7] J. CheEger and D. Ebin, Comparison Theorems in Riemannian Geometry, North Holland, Amsterdam, Oxford, (1975).

[8] R. Bishop And R. CRittenden, Geometry of manifolds, Academic Press, New York, 1964.

Department of Mathematics

Tokyo Institute of TEChNOLOGY

OH-OKayama, Meguro

TOKYO 152, JAPAN 